\title{
Synthesis of long-chained oligo- $\alpha$-aminopyridines by tandem Pd-catalyzed cross-coupling aminations and their helical dinuclear complexes
}

\author{
Hasan Hasanov, ${ }^{\mathrm{a}, \mathrm{b}, \dagger}$ Uan-Kang Tan, ${ }^{\mathrm{b}, \mathrm{c}, \dagger}$ Rui-Ren Wang, ${ }^{\mathrm{a}}$ Gene Hsiang Lee ${ }^{\mathrm{a}}$ and \\ Shie-Ming Peng ${ }^{\mathrm{a}, \mathrm{b}, *}$ \\ ${ }^{a}$ Department of Chemistry, National Taiwan University, Taipei, Taiwan \\ ${ }^{\mathrm{b}}$ Institute of Chemistry, Academia Sinica, Taipei, Taiwan \\ ${ }^{\mathrm{c}}$ Department of Chemical Engineering, Kuang Wu Institute of Technology, Taipei, Taiwan
}

Received 25 June 2004; revised 28 July 2004; accepted 6 August 2004

\begin{abstract}
Three novel multidentate long-chained oligo- $\alpha$-aminopyridine ligands, nonapyridyloctaamine (1, npoa), decapyridylnonaamine (2, dpna), and undecapyridyldecaamine (3, upda) were synthesized successfully by tandem Pd-catalyzed cross-coupling aminations. The helical structures of protonated ligand npoa $\left\{\mathbf{4},\left[\mathrm{H}_{4} \cdot \mathbf{n p o a}\right]\left(\mathrm{SO}_{3} \mathrm{CF}_{3}\right)_{4}\right\}$ and the related dinuclear complexes 5-10 were synthesized and characterized by X-ray diffractions.

(C) 2004 Elsevier Ltd. All rights reserved.
\end{abstract}

Aminopyridines are frequently used as building blocks for synthetic transformations. ${ }^{1-3}$ On the basis of their polynucleating abilities, their derivatives are often studied in organometallic chemistry, ${ }^{4}$ and have industrial applications such as fluorescent dyes. ${ }^{5,6}$ Oligo- $\alpha$-aminopyridines provide multidentate sites with significant flexibility that can produce the helical structures either by intramolecular hydrogen bond formation or self-assembly with metal ions into distinct binding sites. A series of $\alpha$-aminopyridine ligands and various helicates have been reported, such as tetrapyridyltriamine, ${ }^{2}$ pentapyridyltetraamine, ${ }^{2}$ hexapyridylpentaamine, ${ }^{7 a}$ heptapyridylhexaamine, ${ }^{7 \mathrm{~b}}$ and octapyridylheptaamine ${ }^{7 \mathrm{c}}$ from our laboratory, and dinuclear triple helicates from Albrecht's bis-catecholate ligands ${ }^{8}$ and Piguet's bis-terdentate ligands; ${ }^{9}$ dinuclear double helicates from Rice's pyridylthiazole ligands ${ }^{10}$ and Constable's quaterpyridine ligands. ${ }^{11}$ A key to success in assembling these helical aminopyridines is the efficient reaction process from the halopyridines as the starting materials. The preparation of aminopyridines in most studies utilized aromatic nucleophilic substitution $S_{N} A r$, benzyne or $S_{R N} 1$ reac-

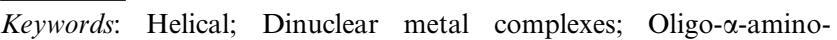
pyridines.

${ }^{*}$ Corresponding author. Tel.: +886 2 23638305; fax: +886 2 23636359; e-mail: smpeng@ntu.edu.tw

${ }^{\dagger}$ These authors contributed equally.
}

tions. ${ }^{12,13}$ These methods are difficult to apply in the consecutive synthesis of polyaminopyridines due to the poor yields, low selectivity from the nucleophilic regiocontrol, the high reaction temperature, and the presence of specific functionality on the heterocyclic rings. ${ }^{12}$ Buchwald and others have recently developed cross-coupling amination to generate mono-aminopyridines starting from their corresponding halopyridines catalyzing with $\operatorname{Pd}(0) /$ bis-phosphine complexes. ${ }^{12,14,15}$ The advantages of Pd-catalyzed $\mathrm{C}-\mathrm{N}$ aminations are mild reaction conditions with high yields; hence this method is utilized to synthesize helical long-chained oligo- $\alpha$-aminopyridines 1-3 effectively.

Oligo- $\alpha$-aminopyridines provide multibinding capabilities with the metal centers, either (i) through nitrogen atoms from both pyridines and amines, ${ }^{16}$ or (ii) only pyridines, ${ }^{17}$ or (iii) only amines, with three different binding modes, syn-syn, anti-anti, and syn-anti (Fig. 1). ${ }^{18}$ These metal complexes are very interesting for

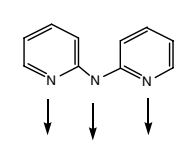

(a)

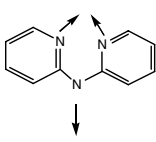

(b)

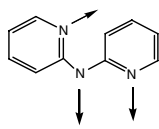

(c)
Figure 1. Types of (a) syn-syn, (b) anti-anti, (c) syn-anti. 
fundamental studies of special physical properties, coordination, ${ }^{19}$ metal-metal interactions, ${ }^{20-25}$ the assembly of helicates, ${ }^{9}$ and biological enzymatic transformations. $^{26-32}$ For example, dinickel and dicopper complexes promote urease $^{30}$ and oxygenase reactions, respectively. ${ }^{31}$ Furthermore, the syn-syn conformation was first found in linear $\mathrm{Cu}_{3}(\mathrm{dpa})_{4} \mathrm{Cl}_{2},{ }^{33}$ anti-anti conformation was observed in $\mathrm{Cu}$ (bipyam) ${ }_{2}{ }^{34}$ and $\mathrm{Cu}$ (mpa) ${ }_{2},{ }^{17}$ and anti-syn conformation was only observed in the dimers of free ligands. ${ }^{33}$ In short, the geometries of the metal helicates are mainly controlled by the partition of binding sites from the ligands while linked to distinct metal centers. It is interesting to assemble long-chained oligo- $\alpha$-aminopyridines, such as nonapyridyloctaamine (1, npoa), decapyridylnonaamine (2, dpna), and undecapyridyldecaamine ( 3 , upda) by employing Buchwald's tandem cross-coupling amination with catalyzation by the $\mathrm{Pd}(0)$ complex/BINAP or DPPP, and their helical dinuclear metal complexes 510. The complexes possess unique features, and have the general formula $\left[\mathrm{M}_{2}(\mathrm{~L})\left(\mathrm{ClO}_{4}\right)_{m}(\mathrm{~S})_{n}\right]\left(\mathrm{ClO}_{4}\right)_{o}$-(solvent $)_{p}$ where $\mathrm{M}=\mathrm{Ni}$ or $\mathrm{Cu}, \mathrm{S}=$ solvent, $m=0-1$, $n=0-3, o=3-4, p=0-5$.

The synthesis of novel long-chained oligo- $\alpha$-aminopyridines 1-3 are shown in Scheme 1 where pyridine was employed as the media to replace the commonly used solvents due to its high solvating ability for the desired adducts, and successful catalyzation of the cross-coupling reaction. ${ }^{18}$

Ligand 1 was obtained via double Pd-catalyzed crosscoupling aminations from (A) and (B), and then subsequent reaction with a half equivalent of 2,6-diaminopyridine. ${ }^{35} \mathrm{~A}$ final yield of $63 \%$ was achieved. Ligand $\mathbf{2}$, in a similar manner, was produced from (A) with an overall $41 \%$ yield via four sequential Pd-catalyzed aminations. ${ }^{36}$

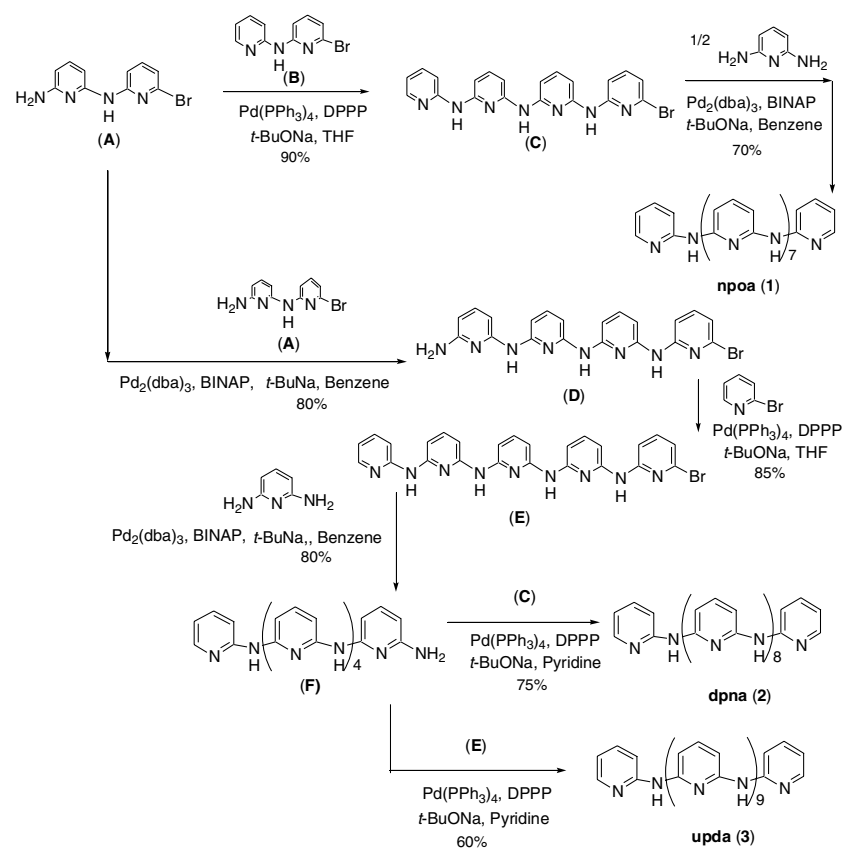

Scheme 1.

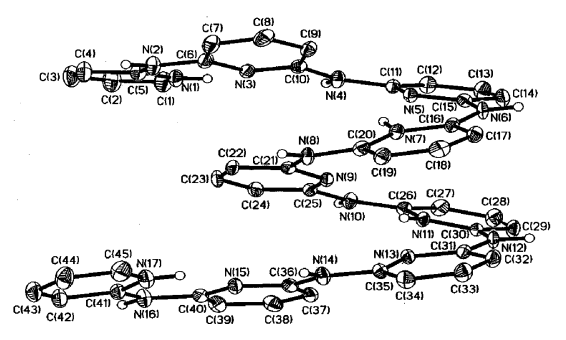

Figure 2. The ORTEP structure of the cationic part in compound 4, thermal ellipsoids $30 \%$ probability level.

Ligand 3 was prepared through a Pd-catalyzed amination from bromo-substrate (E) and amino-substrate (F) in $33 \%$ yield. ${ }^{37}$ The crystal structure $4,{ }^{38}\left[\mathrm{H}_{4} \cdot\right.$ npoa $]-$ $\left(\mathrm{SO}_{3} \mathrm{CF}_{3}\right)_{4}$, a protonated form of ligand $\mathbf{1}$, is shown in Figure 2. It has a highly helical conformation due to its unique intramolecular hydrogen bonds (N1$\mathbf{H} \cdots \mathrm{N} 3, \quad \mathrm{~N} 5-\mathbf{H} \cdots \mathrm{N} 7, \quad \mathrm{~N} 11-\mathbf{H} \cdots \mathrm{N} 9, \quad \mathrm{~N} 11-\mathbf{H} \cdots \mathrm{N} 13$, and N17-H $\cdots$ N15). The TGA data of ligands $\mathbf{1}-\mathbf{3}$ show high thermal stability $\left(\sim 500^{\circ} \mathrm{C}\right)$, which is consistent with Jorgenson's theory. ${ }^{3}$

The dinuclear metal complexes 5-10 (Fig. 4) were prepared by mixing 1 equiv of $\mathrm{Ni}\left(\mathrm{ClO}_{4}\right)_{2}$ or $\mathrm{Cu}\left(\mathrm{ClO}_{4}\right)_{2}$ with 0.55 equiv of synthetic ligands $\mathbf{1}-\mathbf{3}$, respectively, and then growing crystals in the appropriate solvent systems by diffusion techniques. The geometry for both nickel centers demonstrate slightly distorted octahedral in the light blue compound $5\left\{\left[\left(\mathrm{Ni}_{2}\right.\right.\right.$ npoa $\left.)\left(\mathrm{CH}_{3} \mathrm{CN}\right)_{2}\left(\mathrm{H}_{2} \mathrm{O}\right)\right]$ $\left.\left[\mathrm{ClO}_{4}\right]_{3} \cdot(\mathrm{OH}) \cdot\left(\mathrm{H}_{2} \mathrm{O}\right) \cdot\left(\mathrm{CH}_{3} \mathrm{CN}\right)_{4}\right\} \quad$ (Fig. 4), ${ }^{39} \mathrm{~N}(11)-\mathrm{N}-$ (12)-N(13) has a syn-syn conformation, and this molecule has a 'bent-helical' structure (Fig. 3) with approximately 1.5 helical turns, and the full length is around $4.2 \mathrm{~nm}$. The molecular structure of the deep green compound $6\left\{\left[\left(\mathrm{Cu}_{2}\right.\right.\right.$ npoa $\left.)\right]\left[\mathrm{ClO}_{4}\right]_{4} \cdot\left(\mathrm{CH}_{3} \mathrm{CN}\right)_{3}$. $\left(\mathrm{H}_{2} \mathrm{O}\right)_{1.5}$ \}, with a distorted square planar geometry for $\mathrm{Cu}(1)$, and a distorted square pyramidal geometry for $\mathrm{Cu}(2),{ }^{40}$ and a 'regular-helical' structure (Fig. 3) with a syn-anti arrangement of $\mathrm{N}(9)-\mathrm{N}(10)-\mathrm{N}(11)$. This molecule shows more than two helical turns, and its full length is approximately $4.3 \mathrm{~nm}$. Different coordination geometries for two nickel centers are observed in the yellowish-green compound $7\left\{\left[\left(\mathrm{Ni}_{2} \mathbf{d p n a}\right)\left(\mathrm{ClO}_{4}\right)\right]\left[\mathrm{ClO}_{4}\right]_{3}\right.$. $\left.\left(\mathrm{CH}_{3} \mathrm{NO}_{2}\right) \cdot\left(\mathrm{CH}_{3} \mathrm{CN}\right) \cdot\left(\mathrm{C}_{2} \mathrm{H}_{5} \mathrm{OC}_{2} \mathrm{H}_{5}\right)_{2} \cdot\left(\mathrm{CH}_{3} \mathrm{OH}\right)\right\}$ where $\mathrm{Ni}(1)$ exhibits a distorted square pyramid geometry and $\mathrm{Ni}(2)$ has an octahedral shape ${ }^{41}$ The conformation of $\mathrm{N}(7)-\mathrm{N}(8)-\mathrm{N}(9)$ has a syn-syn arrangement, and the whole molecule has a 'bent-helical' structure with approximately two turns, and a length of around $4.8 \mathrm{~nm}$. In the structure of the deep green compound $\mathbf{8}$ $\left\{\left[\left(\mathrm{Cu}_{2} \mathrm{dpna}\right)\right]\left[\mathrm{ClO}_{4}\right]_{4} \cdot\left(\mathrm{CH}_{3} \mathrm{OH}\right)_{2}\right\}$ where both copper ions have distorted square pyramid geometries, ${ }^{42}$ the conformation of $\mathrm{N}(9)-\mathrm{N}(10)-\mathrm{N}(11)$ has a syn-syn geometry, and the whole molecule also has a 'bent-helical' structure with approximately two turns, its full length is around

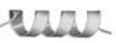

(a) (b)
Figure 3. (a) Regular-helical, (b) bent-helical. 


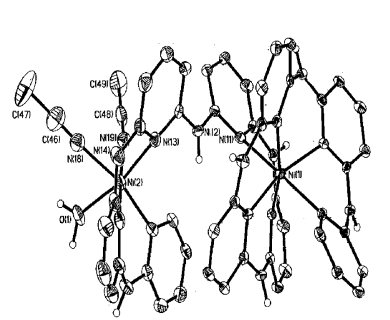

5 (bent)

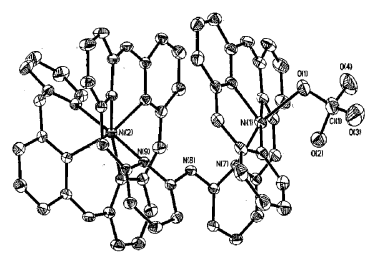

7 (bent)

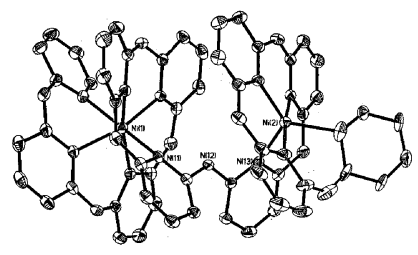

9 (bent)

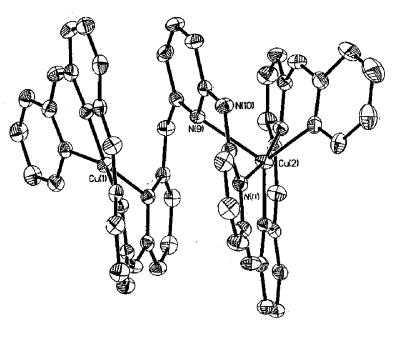

6 (regular)

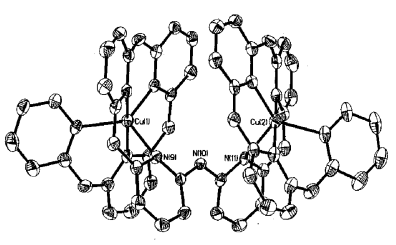

8 (bent)

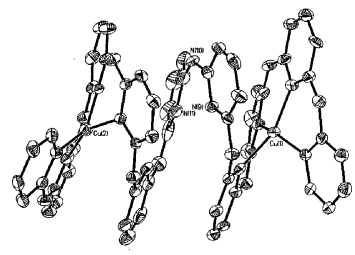

10 (regular)
Figure 4. The ORTEP structure of cationic part of $5\left\{\left[\left(\mathrm{Ni}_{2}-\right.\right.\right.$ npoa) $\left.\left.\left(\mathrm{CH}_{3} \mathrm{CN}\right)_{2}\left(\mathrm{H}_{2} \mathrm{O}\right)\right]\left[\mathrm{ClO}_{4}\right]_{3} \cdot(\mathrm{OH}) \cdot\left(\mathrm{H}_{2} \mathrm{O}\right) \cdot\left(\mathrm{CH}_{3} \mathrm{CN}\right)_{4}, 30 \%\right\}, 6\left\{\left[\left(\mathrm{Cu}_{2^{-}}\right.\right.\right.$ npoa $\left.)]\left[\mathrm{ClO}_{4}\right]_{4} \cdot\left(\mathrm{CH}_{3} \mathrm{CN}\right)_{3} \cdot\left(\mathrm{H}_{2} \mathrm{O}\right)_{1.5}, 50 \%\right\}, 7\left\{\left[\left(\mathrm{Ni}_{2} \mathbf{d p n a}\right)\left(\mathrm{ClO}_{4}\right)\right]\left[\mathrm{ClO}_{4}\right]_{3}\right.$. $\left.\left(\mathrm{CH}_{3} \mathrm{NO}_{2}\right) \cdot\left(\mathrm{CH}_{3} \mathrm{CN}\right) \cdot\left(\mathrm{C}_{2} \mathrm{H}_{5} \mathrm{OC}_{2} \mathrm{H}_{5}\right)_{2} \cdot\left(\mathrm{CH}_{3} \mathrm{OH}\right), 30 \%\right\}, 8\left\{\left[\left(\mathrm{Cu}_{2}\right.\right.\right.$ dpna $\left.)\right]-$ $\left.\left[\mathrm{ClO}_{4}\right]_{4} \cdot\left(\mathrm{CH}_{3} \mathrm{OH}\right)_{2}, \quad 50 \%\right\}, 9\left\{\left[\left(\mathrm{Ni}_{2}\right.\right.\right.$ upda $\left.)\right]\left[\mathrm{ClO}_{4}\right]_{4} \cdot\left(\mathrm{CH}_{3} \mathrm{NO}_{2}\right) \cdot\left(\mathrm{CH}_{3}-\right.$ $\left.\mathrm{OH})_{3.5}, 30 \%\right\}, \mathbf{1 0}\left\{\left[\left(\mathrm{Cu}_{2}\right.\right.\right.$ upda $\left.)\right]\left[\mathrm{ClO}_{4}\right]_{4} \cdot\left(\mathrm{CH}_{2} \mathrm{Cl}_{2}\right)_{0.5} \cdot\left(\mathrm{CH}_{3} \mathrm{NO}_{2}\right)_{4} \cdot\left(\mathrm{H}_{2} \mathrm{O}\right)_{3}$, $20 \%$ thermal ellipsoids probability level\}.

$4.6 \mathrm{~nm}$. The geometry for the nickel ions in the yellowish-orange compound $\mathbf{9},\left\{\left[\left(\mathrm{Ni}_{2} \mathbf{u p d a}\right)\right]\left[\mathrm{ClO}_{4}\right]_{4} \cdot\left(\mathrm{CH}_{3}\right.\right.$ $\left.\left.\mathrm{NO}_{2}\right) \cdot\left(\mathrm{CH}_{3} \mathrm{OH}\right)_{3.5}\right\}$ where $\mathrm{Ni}(1)$ has a distorted square pyramid geometry and $\mathrm{Ni}(2)$ exhibits an octahedral shape; ${ }^{43} \mathrm{~N}(11)-\mathrm{N}(12)-\mathrm{N}(13)$ has a syn-syn conformation, and the whole molecule has a 'bent-helical' structure with more than two turns, its rough length is around $5.2 \mathrm{~nm}$. Both copper ions have distorted square planer shapes (Fig. 4) ${ }^{44}$ in the deep green compound $10\left\{\left[\left(\mathrm{Cu}_{2}\right.\right.\right.$ upda $\left.\left.)\right]\left[\mathrm{ClO}_{4}\right]_{4} \cdot\left(\mathrm{CH}_{2} \mathrm{Cl}_{2}\right)_{0.5} \cdot\left(\mathrm{CH}_{3} \mathrm{NO}_{2}\right)_{4} \cdot\left(\mathrm{H}_{2} \mathrm{O}\right)_{3}\right\}$, and all the nitrogen linkage arrangements have anti-anti conformations, the whole molecule has a 'regular-helical' structure with more than three complete turns, and its rough length is around $5.8 \mathrm{~nm}$.

The magnetic properties were measured using ground powders of the dinuclear compounds 5-10 $\mathbf{1 0}^{35-40}$ (at $10 \mathrm{kG})$, and a simple spin-only model of magnetic moment $\left(\mu_{\mathrm{eff}}\right)^{45}$ was adapted due to their long metalmetal distances. The $\mu_{\mathrm{eff}}=\left[n_{1}\left(n_{1}+2\right)+n_{2}\left(n_{2}+2\right)\right]^{1 / 2}$, where $n_{1}$ represents the unpaired electrons of metal (1), and $n_{2}$ represents the unpaired electrons of metal (2), was calculated. Dinickel compounds 5, 7, and 9 have $\mu_{\mathrm{eff}}=3.9,4.0$, and 4.2 , respectively, that are close to the high spin predicted value, $\mu_{\text {eff }}=[2(2+2)+2(2+$ $2)]^{1 / 2}=4.0$. The dicopper compounds $\mathbf{6}, \mathbf{8}$, and $\mathbf{1 0}$ all have $\mu_{\mathrm{eff}}=2.3$, which agrees with the predicted value $\mu_{\mathrm{eff}}=[1(1+2)+1(1+2)]^{1 / 2}=2.4$. The magnetic studies have revealed that the dinickel complexes $(\mathbf{5}, \mathbf{7}$, and 9) exhibit a slight antiferromagnetic behavior at temperature $<50 \mathrm{~K}$, and paramagnetic properties at all other tested temperatures. The dicopper complexes $(\mathbf{6}, \mathbf{8}$, and 10) show paramagnetic behaviors at all recorded temperatures. This indicates that these magnetic behaviors for the dinuclear complexes are actually similar to their corresponding mononuclear complexes. The paramagnetic behaviors are also consistent with the NMR observations where the downfield peaks occur at $10-20 \mathrm{ppm}$ for metal complexes versus their corresponding free ligands.

This work reports the effective synthesis of novel helical oligo- $\alpha$-aminopyridine ligands $\mathbf{1}-\mathbf{3}$ by tandem Pd-catalyzed cross-coupling amination, and their corresponding dinuclear $\left(\mathrm{Ni}^{2+} / \mathrm{Ni}^{2+}\right.$ and $\left.\mathrm{Cu}^{2+} / \mathrm{Cu}^{2+}\right)$ metal complexes 5-10. Ligands 1-3 show coordination to the metal centers only through the nitrogen atoms on the pyridine rings. Only dinuclear complexes $\mathbf{6}$ and $\mathbf{1 0}$ show 'regular-helical' structures and with weak $\pi-\pi$ interactions. It is worthwhile to note that the dinuclear complexes 5-10 exhibit the longest metal-metal distances that have been reported for complexes with oligo- $\alpha$-aminopyridine ligands, with distances between the metal centers of $6.042,4.639,6.231,5.836,5.900$, and $7.420 \AA$, respectively.

Crystallographic data for the structural analysis have been registered in the Cambridge Crystallographic Data Center: compound $\mathbf{4}$ was registered as CCDC No. 201554, compound 5 as CCDC No. 201556, compound 6 as CCDC No. 201555, compound 7 as CCDC No. 201558, compound 8 as CCDC No. 201557, compound 9 as CCDC No. 201560, and compound $\mathbf{1 0}$ as CCDC No. 201559. Information may be obtained free of charge from the Director, CCDC, 12 Union Road, Cambridge, CB2 1EZ, UK (fax: +44-1223-336033; e-mail: deposit@ccdc.cam.ac.uk or http://www.ccdc.cam.ac.uk).

\section{Acknowledgements}

We thank the National Science Council and the Ministry of Education of the Republic of China for the financial supports.

\section{References and notes}

1. Textbook of Organic Medicinal and Pharmaceutical Chemistry; Delgado, J. N., Gisvold, O., Remers, W. A., Eds.; Lippincott-Raven: Philadelphia, PA, 1998; p 666.

2. Peng, C.-H.; Wang, C.-C.; Lee, H.-C.; Lo, W.-C.; Lee, G.-H.; Peng, S.-M. J. Chin. Chem. Soc. (Taipei) 2001, 48, 987-996.

3. Leung, M.-K.; Mandal, A. B.; Wang, C. C.; Lee, G.-H.; Peng, S.-M.; Cheng, H.-L.; Her, G.-R.; Chao, I.; Lu, H.-F.; Sun, Y.-C.; Shiao, M.-Y.; Chou, P.-T. J. Am. Chem. Soc. 2002, 124, 4287-4297.

4. (a) Yang, M.-H.; Lin, T.-W.; Chou, C.-C.; Lee, H.-C.; Chang, H.-C.; Lee, G.-H.; Leung, M.-K.; Peng, S.-M. 
J. Chem. Soc., Chem. Commun. 1997, 2279-2280; (b) Lai, S.-Y.; Lin, T.-W.; Chen, Y.-H.; Wang, C.-C.; Lee, G.-H.; Yang, M.-H.; Leung, M.-K.; Peng, S.-M. J. Am. Chem. Soc. 1999, 121, 250-251; (c) Chang, H.-C.; Li, J.-T.; Wang, C.-C.; Lin, T.-W.; Lee, H.-C.; Lee, G.-H.; Peng, S.-M. Eur. J. Inorg. Chem. 1999, 1243-1251; (d) Basuli, F.; Peng, S.-M.; Bhattacharya, S. Inorg. Chem. 2001, 40, 1126-1133.

5. Araki, K.; Mutai, T.; Shigemitsu, Y.; Yamada, M.; Nakajima, T.; Kuroda, S.; Shimao, I. J. Chem. Soc., Perkin. Trans. 2 1996, 613-617.

6. Sathyamoorthy, G.; Soong, M. L.; Ross, T. W.; Boyer, J. H. Heteroatom. Chem. 1993, 4, 603-608.

7. (a) Lee, C.-C. Master Thesis, Taiwan University, May, 2000; pp 26-42; (b) Lin, Y.-S. Master Thesis, Taiwan University, May, 2000; pp 29-42; (c) Unpublished laboratory data.

8. Albrecht, M. Chem. Soc. Rev. 1998, 27, 281-287.

9. Elhabiri, M.; Scopelliti, R.; Bunzli, J.-C. G.; Piguet, C. J. Am. Chem. Soc. 1999, 121, 10747-10762.

10. (a) Rice, C. R.; Wörl, S.; Jeffery, J. C.; Paul, R. L.; Ward, M. D. J. Chem. Soc., Dalton. Trans. 2001, 550-559; (b) Rice, C. R.; Wörl, S.; Jeffery, J. C.; Paul, R. L.; Ward, M. D. J. Chem. Soc., Chem. Commun. 2000, 23, 15291530 .

11. Constable, E. C.; Elder, S. M.; Hannon, M. J.; Martin, A.; Raithby, P. R.; Tocher, D. A. J. Chem. Soc., Dalton. Trans. 1996, 2423-2433.

12. (a) Miyazaki, Y.; Kanbara, T.; Yamamoto, T. Tetrahedron Lett. 2002, 43, 7945-7948; (b) Kosugi, M.; Kaweyama, M.; Migita, T. Chem. Lett. 1983, 927-933; (c) Louie, J.; Hartwig, J. F. Tetrahedron Lett. 1995, 36, 3609-3612; (d) Hartwig, J. F. Angew. Chem., Int. Ed. 1998, 37, 20462067.

13. March, J. Advanced Organic Chemistry; John Wiley and Sons: New York, NY, 1985; 576.

14. Wagaw, S.; Buckwald, S. L. J. Org. Chem. 1996, 61, 72407241.

15. Wolfe, J.; Buckwald, S. L. J. Org. Chem. 2000, 65, 1144 1157.

16. Cotton, F. A.; Daniels, L. M.; Murillo, C. A.; Pascual, I.; Zhou, H.-C. J. Am. Chem. Soc. 1999, 121, 68566861.

17. Baxter, C. E.; Rodig, O. R.; Schlatzer, R. K.; Sinn, E. Inorg. Chem. 1979, 18, 1918-1921.

18. Hasanov, H.; Tan, U.-K.; Lin, Y.-S.; Lee, C.-C.; Lee, G.-H.; Lin, T.-W.; Peng, S.-M. Inorg. Chim. Acta 2003, 351, 369-377.

19. Lai, S.-Y.; Wang, C.-C.; Chen, Y.-H.; Lee, C.-C.; Liu, Y.-H.; Peng, S.-M. J. Chin. Chem. Soc. (Taipei) 1999, 46, $477-485$.

20. Cotton, F. A.; Czuchajowska, J.; Feng, X. Inorg. Chem. 1990, 29, 4329-4335.

21. Wu, L. P.; Field, P.; Morrisey, T.; Murphy, C.; Nagle, P.; Hathaway, B.; Simmons, C.; Thornton, P. J. Chem. Soc., Dalton. Trans. 1990, 3835-3840.

22. Pyrka, G. J.; El-Mekki, M.; Prinkerton, A. A. J. Chem. Soc., Chem. Commun. 1991, 84-85.

23. Aduldecha, A.; Hathaway, B. J. Chem. Soc., Dalton. Trans. 1991, 993-998.

24. (a) Cotton, F. A.; Daniels, L. M.; Murillo, C. A.; Pascual, I. J. Am. Chem. Soc. 1997, 119, 10223-10224; (b) Cotton, F. A.; Daniels, L. M.; Murillo, C. A.; Wang, X. P. J. Chem. Soc., Chem. Commun. 1998, 23, 3940.

25. Sheu, J.-T.; Liu, C.-C.; Chao, I.; Wang, C.-C.; Peng, S.-M. J. Chem. Soc., Chem. Commun. 1996, 23, 315-316.

26. Poyner, R. R.; Reed, G. H. Biochemistry 1992, 31, 71667173.
27. Reczkowski, R. S.; Ash, D. E. J. Am. Chem. Soc. 1992 , 114, 10992-10994.

28. Vincent, J. B.; Crowder, M. W.; Averill, B. A. Trends Biochem. Sci. 1992, 17, 105-110.

29. Roderick, S. L.; Matthews, B. W. Biochemistry 1993, 32, 3907-3912.

30. Day, E. P.; Peterson, J.; Sendova, M. S.; Todd, M. J.; Hausinger, R. P. Inorg. Chem. 1993, 32, 634-638.

31. Karlin, K. D.; Hayes, J. C.; Gultneh, Y.; Cruse, R. W.; McKown, J. W.; Hutchinson, J. P.; Zubieta, J. J. Am. Chem. Soc. 1984, 106, 2121-2128.

32. (a) Burley, S. K.; Daivid, P. R.; Taylor, A.; Lipscomb, W. N. Proc. Natl. Acad. Sci. U.S.A. 1990, 87, 6878-6882; (b) Burley, S. K.; David, P. R.; Lipscomb, W. N. Proc. Natl. Acad. Sci. U.S.A. 1991, 88, 6916-6920.

33. Yang, E.-C.; Cheng, M.-C.; Tsai, M.-S.; Peng, S.-M. J. Chem. Soc., Chem. Commun. 1994, 2377-2378.

34. Rodig, O. R.; Brueckner, T.; Huriburt, B. R.; Schlatzer, R. K.; Benable, T. L.; Sinn, E. J. Chem. Soc., Chem. Commun. 1981, 196-198.

35. npoa, nonapyridyloctaamine, 1: Anal. Calcd for $\mathrm{C}_{45} \mathrm{H}_{37} \mathrm{~N}_{17}$ : $\mathrm{C}$ 66.25, H 4.57, N 29.18. Found: C 66.36, H 4.56, N 29.08. IR (KBr): 3408, 3310, 3205, 3021, 1674, $1582,1431,1307,1149,1774,1149,715 \mathrm{~cm}^{-1}$. ${ }^{1} \mathrm{H} \mathrm{NMR}$ $\left(400 \mathrm{MHz}, \mathrm{DMSO}-d_{6}\right): \delta 9.38(\mathrm{~s}, 2 \mathrm{H}, \mathrm{NH}), 9.09(\mathrm{~s}, 6 \mathrm{H}$, $\mathrm{NH}), 6.83-8.21(\mathrm{~m}, 29 \mathrm{H}$, aromatic $\mathrm{H})$. FAB/MS $[\mathrm{m} / \mathrm{z}]$ : [816 (M) $\left.{ }^{+}\right]$.

36. dpna, decapyridylnonaamine, 2: Anal. Calcd for $\mathrm{C}_{50} \mathrm{H}_{41} \mathrm{~N}_{19}$ : C 66.14, $\mathrm{H}$ 4.55, N 29.31. Found: C 66.43, H 4.42, N 29.15. IR (KBr): 3448, 3310, 3198, 1635, 1562, $1516,1418,1352,1155 \mathrm{~cm}^{-1}$. ${ }^{1} \mathrm{H}$ NMR (400 MHz, DMSO$\left.d_{6}\right): \delta 8.49(\mathrm{~s}, 2 \mathrm{H}, \mathrm{NH}), 8.20(\mathrm{~s}, 7 \mathrm{H}, \mathrm{NH}), 5.99-7.35(\mathrm{~m}$, $32 \mathrm{H}$, aromatic $\mathrm{H})$. FAB/MS [m/z]: [908 $\left.(\mathbf{M})^{+}\right]$.

37. upda, undecapyridyldecaamine, 3: Anal. Calcd for $\mathrm{C}_{55} \mathrm{H}_{45} \mathrm{~N}_{21}$ : C 66.05, H 4.54, N 29.41. Found: C 66.30, H 4.38, N 29.32. IR (KBr): 3416, 3204, 2952, 1587, 1514, 1434, 1308, $778 \mathrm{~cm}^{-1}$. ${ }^{1} \mathrm{H}$ NMR (400 MHz, DMSO-d $\left.d_{6}\right): \delta$ $9.36(\mathrm{~s}, 2 \mathrm{H}, \mathrm{NH}), 9.06(\mathrm{~s}, 8 \mathrm{H}, \mathrm{NH}), 6.84-8.20(\mathrm{~m}, 35 \mathrm{H}$, aromatic H). FAB/MS $[\mathrm{m} / \mathrm{z}]$ : $\left[1000(\mathrm{M})^{+}\right]$.

38. Crystallographic data for 4: $\mathrm{C}_{52} \mathrm{H}_{45.50} \mathrm{~F}_{12} \mathrm{~N}_{18.50} \mathrm{O}_{12} \mathrm{~S}_{4}$, $M=1477.81$, monoclinic, space group $C_{2} / c, a=26.5696$ (11), $b=14.6662$ (6), $c=33.9125$ (14), $\alpha=90^{\circ}, \quad \beta=$ $111.3060^{\circ}(10), \gamma=90^{\circ}, \quad V=12311.7 \quad(9) \AA^{3}, \quad Z=8$, $D_{\text {calcd }}=1.595 \mathrm{~g} / \mathrm{cm}^{3}, \mu\left(\mathrm{MoK}_{\alpha}\right)=0.268 \mathrm{~mm}^{-1}, \lambda=0.71073 \AA$, $T=150 \quad$ (1) $\mathrm{K}, \quad \theta=1.29-26.43^{\circ}, \quad T_{\min } / T_{\max }=0.8562 /$ 0.9280 , independent reflns. $=12606 \quad\left(R_{\text {int }}=0.0850\right)$, $R_{\mathrm{f}}=0.0549, \mathrm{GOF}=1.059$. The structure was solved in Bruker SMART by direct method and expanded by using Fourier techniques, the functions were minimized during least-squares cycles where $R=\Sigma|| \mathrm{F}_{0}|-| \mathrm{F}_{\mathrm{c}}|| / \Sigma\left|\mathrm{F}_{0}\right|$; $\left(F_{0}^{2}\right)=\left[\Sigma\left\{w\left(F_{0}^{2}-F_{\mathrm{c}}^{2}\right)^{2}\right\} / \Sigma\left\{w\left(F_{0}^{2}\right)^{2}\right\}\right]^{1 / 2}$. The nonhydrogen atoms were refined anisotropically.

39. Crystallographic data for 5: $\mathrm{C}_{57} \mathrm{H}_{60} \mathrm{Cl}_{3} \mathrm{~N}_{23} \mathrm{Ni}_{2} \mathrm{O}_{15}, M=$ 1531.05 , triclinic, space group $P \overline{1}, a=11.8920$ (6), $b=$ 13.8128 (7), $c=22.5784$ (12), $\alpha=83.140^{\circ}$ (1), $\beta=83.697^{\circ}$ (1), $\gamma=86.049^{\circ}(1), V=1852.83(3) \AA^{3}, Z=2, D_{\text {calcd }}=$ $1.392 \mathrm{~g} / \mathrm{cm}^{3}, \quad \mu\left(\mathrm{MoK}_{\alpha}\right)=0.701 \mathrm{~mm}^{-1}, \quad \lambda=0.71073 \AA$, $T=150$ (1) K, $\theta=0.91-26.37^{\circ}, \quad T_{\min } / T_{\max }=0.6904 /$ 0.8621 , independent reflns. $=14936\left(R_{\text {int }}=0.0881\right) . R_{\mathrm{f}}=$ $0.0896, \mathrm{GOF}=1.024$. The structure was solved in Bruker SMART with the same method described previously.

40. Crystallographic data for 6: $\mathrm{C}_{51} \mathrm{H}_{49} \mathrm{Cl}_{4} \mathrm{~N}_{20} \mathrm{Cu}_{2} \mathrm{O}_{17.50}$, $M=1490.98$, monoclinic, space group $P 2{ }_{1} / c, a=22.3833$ (12), $b=13.1494$ (7), $c=20.6251 \quad(11), \quad \alpha=90^{\circ}, \quad \beta=$ $101.995^{\circ}$ (1), $\gamma=90^{\circ}, V=5938.0(5) \AA^{3}, Z=4, D_{\text {calcd }}=$ $1.668 \mathrm{~g} / \mathrm{cm}^{3}, \quad \mu\left(\mathrm{MoK}_{\alpha}\right)=0.987 \mathrm{~mm}^{-1}, \quad \lambda=0.71073 \AA$, $T=150 \quad$ (1) $\mathrm{K}, \theta=2.43-27.50^{\circ}, \quad T_{\min } / T_{\max }=0.7154 /$ 0.8311 , independent reflns. $=13624 \quad\left(R_{\text {int }}=0.0331\right)$. $R_{\mathrm{f}}=0.0451, \mathrm{GOF}=1.014$. The structure was solved in 
Bruker SMART with the same method described previously.

41. Crystallographic data for 7: $\mathrm{C}_{62} \mathrm{H}_{71} \mathrm{Cl}_{4} \mathrm{~N}_{21} \mathrm{Ni}_{2} \mathrm{O}_{21}, M=$ 1705.62, triclinic, space group $P \overline{1}, a=14.6520$ (1), $b=$ 15.4574 (1), $c=17.5339$ (2), $\alpha=100.7550^{\circ}$ (4), $\beta=$ $98.3837^{\circ}$ (4), $\gamma=110.4784^{\circ}$ (4), $V=3557.37 \quad(5) \mathrm{AA}^{3}$, $Z=2, D_{\text {ocalcd }}=1.592 \mathrm{~g} / \mathrm{cm}^{3}, \mu\left(\mathrm{MoK}_{\alpha}\right)=0.770 \mathrm{~mm}^{-1}, \lambda=$ $0.71073 \AA, T=150$ (1) $\mathrm{K}, \theta=2.43-27.50^{\circ}, T_{\min } / T_{\max }=$ $0.774 / 0.942$, independent reflns. $=16291\left(R_{\mathrm{int}}=0.0662\right)$. $R_{\mathrm{f}}=0.0665, \mathrm{GOF}=1.021$. The structure was solved in Nonius KappaCCD with the same method described previously.

42. Crystallographic data for 8: $\mathrm{C}_{52} \mathrm{H}_{49} \mathrm{Cl}_{4} \mathrm{~N}_{19} \mathrm{Cu}_{2} \mathrm{O}_{18}$, $M=1496.98$, monoclinic, space group $P 2_{1} / c, a=15.4476$ (3), $b=14.4702$ (2), $c=25.9158$ (6), $\alpha=90^{\circ}, \beta=98.3837^{\circ}$ (4), $\gamma=90^{\circ}, V=5735.39(19) \AA^{3}, Z=4, D_{\text {calcd }}=1.734 \mathrm{~g} /$ $\mathrm{cm}^{3}, \mu\left(\mathrm{MoK}_{\alpha}\right)=1.022 \mathrm{~mm}^{-1}, \lambda=0.71073 \AA, T=150$ (1) $\mathrm{K}, \theta=1.92-25^{\circ}, T_{\min } / T_{\max }=0.5449 / 0.8950$, independent reflns. $=10088\left(R_{\text {int }}=0.0768\right) . R_{\mathrm{f}}=0.0546$, GOF $=1.051$. The structure was solved in Nonius KappaCCD with the same method described previously.
43. Crystallographic data for 9: $\mathrm{C}_{59.50} \mathrm{H}_{62} \mathrm{Cl}_{4} \mathrm{~N}_{22} \mathrm{Ni}_{2} \mathrm{O}_{21.50}$, $M=1688.53$, monoclinic, space group $P 2_{1} / n, a=11.0634$ (1), $b=24.8780$ (2), $c=25.0143$ (2), $\alpha=90^{\circ}, \beta=101.0158^{\circ}$ (3) $, \gamma=90^{\circ}, V=6757.96(10) \AA^{3}, Z=4, D_{\text {calcd }}=1.660 \mathrm{~g} /$ $\mathrm{cm}^{3}, \mu\left(\mathrm{MoK}_{\alpha}\right)=0.811 \mathrm{~mm}^{-1}, \lambda=0.71073 \AA, T=150(1)$ $\mathrm{K}, \theta=1.84-27.50^{\circ}, T_{\min } / T_{\max }=0.646 / 0.931$, independent reflns. $=15455\left(R_{\text {int }}=0.0581\right) . R_{\mathrm{f}}=0.0826$, GOF $=1.040$. The structure was solved in Nonius KappaCCD with the same method described previously.

44. Crystallographic data for 10: $\mathrm{C}_{59.50} \mathrm{H}_{64} \mathrm{Cl}_{5} \mathrm{~N}_{25} \mathrm{Cu}_{2} \mathrm{O}_{27}$, $M=1865.69$, monoclinic, space group $P 2_{1} / n, a=11.6942$ (2), $b=26.4115$ (5), $c=25.0251 \quad(5), \alpha=90^{\circ}$ (1), $\beta=$ $99.730^{\circ}$ (1), $\gamma=90^{\circ}, V=7618.1(2) \AA^{3}, \quad Z=4, D_{\text {calcd }}=$ $1.627 \mathrm{~g} / \mathrm{cm}^{3}, \mu\left(\mathrm{MoK}_{\alpha}\right)=0.832 \mathrm{~mm}^{-1}, \lambda=0.71073 \AA, T=$ 150 (1) $\mathrm{K}, \theta=2.07-25^{\circ}, T_{\min } / T_{\max }=0.830 / 0.974$, independent reflns. $=13385 \quad\left(R_{\mathrm{int}}=0.0747\right) . \quad R_{\mathrm{f}}=0.1624$, $\mathrm{GOF}=1.058$. The structure was solved in Nonius KappaCCD with the same method described previously.

45. Teweldemedhin, Z. S.; Fuller, R. L.; Greenblatt, M. J. Chem. Edu. 1996, 73, 906-909. 